\title{
Tilt from a head-inverted position produces displacement of visual subjective vertical in the opposite direction
}

\author{
DONALD E. PARKER and RICK L. POSTON \\ Miami University, Oxford, Ohio
}

\begin{abstract}
Observers who lie supine with their heads inverted report large (up to $60^{\circ}$ ) tilt of a light line in an otherwise dark room when their heads and/or bodies are tilted. Most observers report that visual subjective vertical is tilted in the direction opposite to the head/body tilt. The results can be interpreted by employing a model developed by Mittelstaedt (1983), which suggests that visual subjective vertical is derived from a gravity vector transduced by vestibular and somesthetic receptors combined with "idiotropic vectors" that represent the orientation of the observer's own head and body axes.
\end{abstract}

Suppose an observer lies supine on a table with his head hanging over the edge. If he looks at a truly vertical light line in an otherwise dark room while he tilts his head, he usually will report rotation of the light line in the same direction as the head tilt. When asked to align the line with gravity, he will then rotate it in the direction opposite to the head tilt to compensate for the illusory rotation.

Investigation of errors made by observers when they attempt to orient a light line to gravitational vertical while in a darkened room and while the head and body are tilted is interesting because it suggests how signals from vestibular and somesthetic receptors that indicate head/body orientation with respect to gravity are combined with information representing egocentric orientation of the head and body to yield perceived orientation of the line on the retina. The specific phenomenon of displacement of subjective visual vertical produced by tilts from a headinverted position is interesting because large displacements result from small head tilts and because most observers perceive subjective vertical to be tilted in the direction opposite to their head/body tilt.

The effects of body tilt on visual subjective vertical have been examined in numerous investigations since Aubert first reported that a vertical streak of light tilted in the opposite direction when he tilted his head from an upright position to one side. In these investigations the observer usually has been tilted around the ventral-dorsal body axis, starting from an upright position. After being placed in a particular position, the observer is asked to

This work was supported by Contract NAS 9-14538 between Miami University and the National Aeronautics and Space Administration. We thank $\mathrm{H}$. Mittelstaedt, who suggested the variation of his model presented in Figure 4, and A. Pantle and S. Berry for their comments. Our mail ing address is: Department of Psychology, Miami University, Oxford, OH 45056 . rotate a luminous line until it appears vertical. Differences between the line settings and gravitational vertical (errors) are recorded. Relatively small errors in the direction opposite to the head/body tilt usually are obtained following tilts up to about $60^{\circ}$ ("E effect"). For tilts beyond $60^{\circ}$, observers place the luminous line increasingly in the direction of their own tilt ("A effect"). Errors become most pronounced at tilts of $150^{\circ}$, where the observers' line settings may deviate from true vertical by more than $50^{\circ}$ (see Howard, 1982).

Previously, the effects of body tilt on visual subjective vertical have been interpreted in terms of a "compensation" principle or as indicative of decreased vestibular otolith receptor sensitivity. Some investigators have proposed that otolith and somesthetic inputs compensate for the disturbance associated with rotation of the image away from the normally vertical meridian of the retina (Bischof, 1974; Parker, 1980; Parker, Poston, \& Gulledge, 1983). The large errors in setting a line to gravity associated with large $\left(+60^{\circ}\right)$ body tilts have been interpreted as undercompensation, that is, as a failure of the otolith and somesthetic receptors to provide adequate compensation for the rotation of the image. Schone and his colleagues (Schone, 1964, 1965; Udo de Haes \& Schone, 1970) noted the increased variability of judgments of the visual vertical when the head was tilted more than $60^{\circ}$, and suggested that this indicates decreasing sensitivity of the otolith receptors at these positions.

In contrast to the usual procedure, we examined the effects of small head/body tilts starting from a head-inverted position, and were surprised to note that these tilts produced large, consistent apparent tilts of a vertical line. Ideas of undercompensation and reduced sensitivity did not appear adequate to explain these initial observations; therefore, we undertook three experiments to explore this phenomenon further. 


\section{EXPERIMENT 1}

\section{Method}

Four observers, none of whom reported a history of auditory or vestibular difficulties, participated in Experiment 1.

The observers were placed in an aluminum cylinder that enclosed a styrofoam body mold (Parker, Wood, Gulledge, \& Goodrich, 1979). Their heads were dorsal-flexed and restrained by adjustable ear pads attached to the cylinder (Figure 1). The entire cylinder could be rotated to a desired head/body tilt position. Alternatively, the pads could be adjusted to tilt the head, leaving the body straight, or tilt the body while the head remained straight.

The visual target was a set of seven colinear dots plotted on a video monitor. The visual angle subtended by the line of dots was $19^{\circ}$. The observer aligned the line to apparent vertical using a handheld control. Presentation of the stimulus line and recording of the observer's settings were under microprocessor control. Only the line of dots was visible to the observer.

The observers were instructed to align the line of dots with gravitational vertical. They were told to imagine that the bottom dot was a weight on a plumb line and given practice in setting the line in the light to ensure that they understood the instructions.

Each observer set the line to his/her perception of vertical (subjective vertical) while the head was inverted (straight), tilted $30^{\circ}$ left, or tilted $30^{\circ}$ right (Figure 1). Ten settings were made in each position at 1 -min intervals after a 1 -min stabilization period. A session consisted of 10 settings in each of the three positions. All ob-

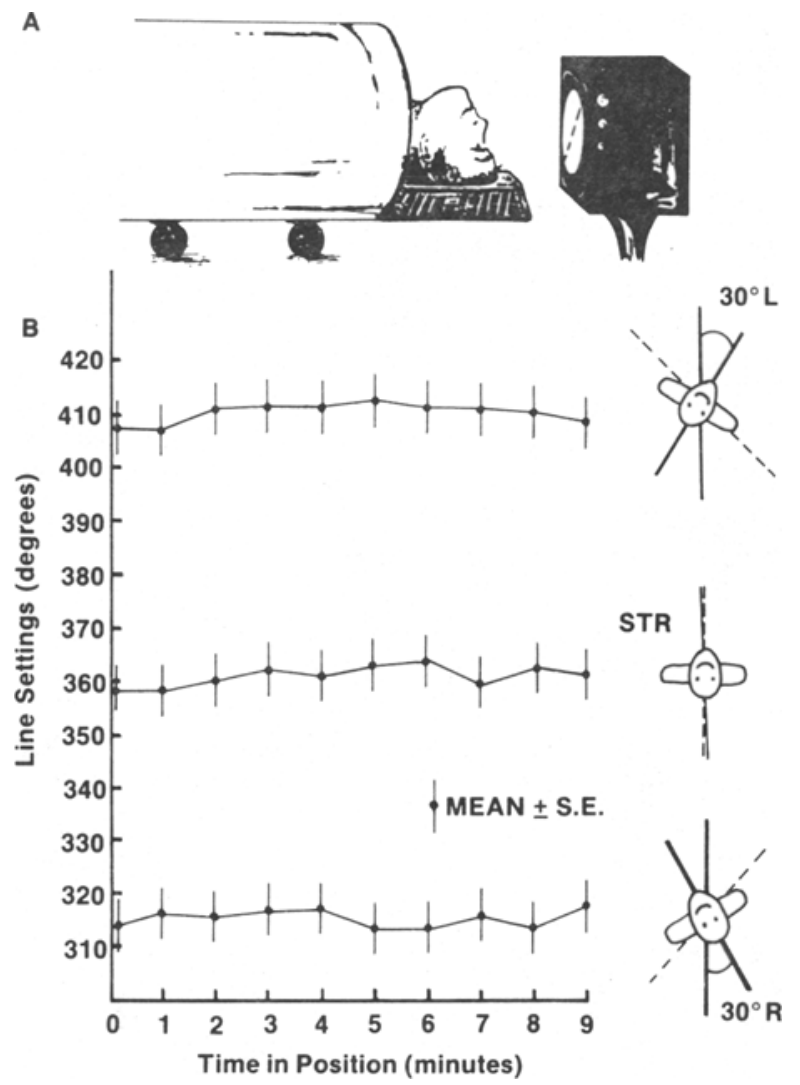

Figure 1. Average line settings to visual subjective vertical from four observers during $10 \mathrm{~min}$ in straight (head inverted), $30^{\circ}$ lefttilt, and $30^{\circ}$ right-tilt positions. Head tilt resulted in displacement of subjective vertical (dashed line) in the direction opposite to the tilt. Settings to subjective vertical changed little across the observation period. servers completed six sessions separated by at least $24 \mathrm{~h}$. Order of positions was counterbalanced.

\section{Results}

The observers' settings to subjective vertical were in the direction opposite to the head tilt, and the average signed errors of the settings across observers and time were $50^{\circ}$ for the left-tilt position, $45^{\circ}$ for the right-tilt position, and $1^{\circ}$ for the straight position (Figure 1). Differences in line settings as a function of head-body position were highly significant $[\mathrm{F}(2,6)=23.4, \mathrm{p}<.002]$. As illustrated in Figure 1, the settings changed little across the $10 \mathrm{~min}$ of observation. Analysis of variance indicated that a very small portion of the variability in the settings was associated with time in position $[\mathrm{F}(9,27)=1.11$, $\mathrm{p}>.4]$.

The results of the first experiment confirmed preliminary studies indicating that tilt from a head-inverted position produced displacement of subjective vertical in the direction opposite to the head tilt. The second experiment was designed to assess individual differences revealed in both the preliminary studies and the first experiment.

\section{EXPERIMENT 2}

\section{Method}

In the second experiment, 50 undergraduate students set the line to their subjective vertical while in each of the three positions used in the first experiment. Line settings were obtained at 1-min intervals after a 1-min stabilization period. A session consisted of four settings in each of the three head/body positions. Position order was counterbalanced across observers and each observer completed two sessions separated by at least $24 \mathrm{~h}$.

\section{Results}

Figure 2 illustrates the relative frequency of line settings averaged across the two sessions for each of the three head/body positions. The curves indicate that most observers reported that their subjective vertical was displaced in the direction opposite to the head/body tilt; however, some observers' settings to vertical were unchanged by tilt and others reported that their subjective vertical was displaced in the same direction as the head/body tilt. Clearly, individuals differ in their responses to tilt from a head-inverted position.

Analysis of variance indicated that line settings varied as a function of head/body position $[\mathrm{F}(2,96)=98.4, \mathrm{p}<$ $.0001]$ but that neither time $[F(3,144)=0.88, p>.45]$ nor session $[F(1,45)=0.12, p>.72]$ accounted for a significant portion of the variability in the settings. Overall, observers' reports of subjective vertical again were in the direction opposite to head/body tilt, and the average errors in the settings across observers and time were $35^{\circ}$ for the left-tilt position, $37^{\circ}$ for the right-tilt position, and $2^{\circ}$ for the straight position.

\section{EXPERIMENT 3}

The third experiment was designed to determine the relative contributions of the head and body to the tilt- 


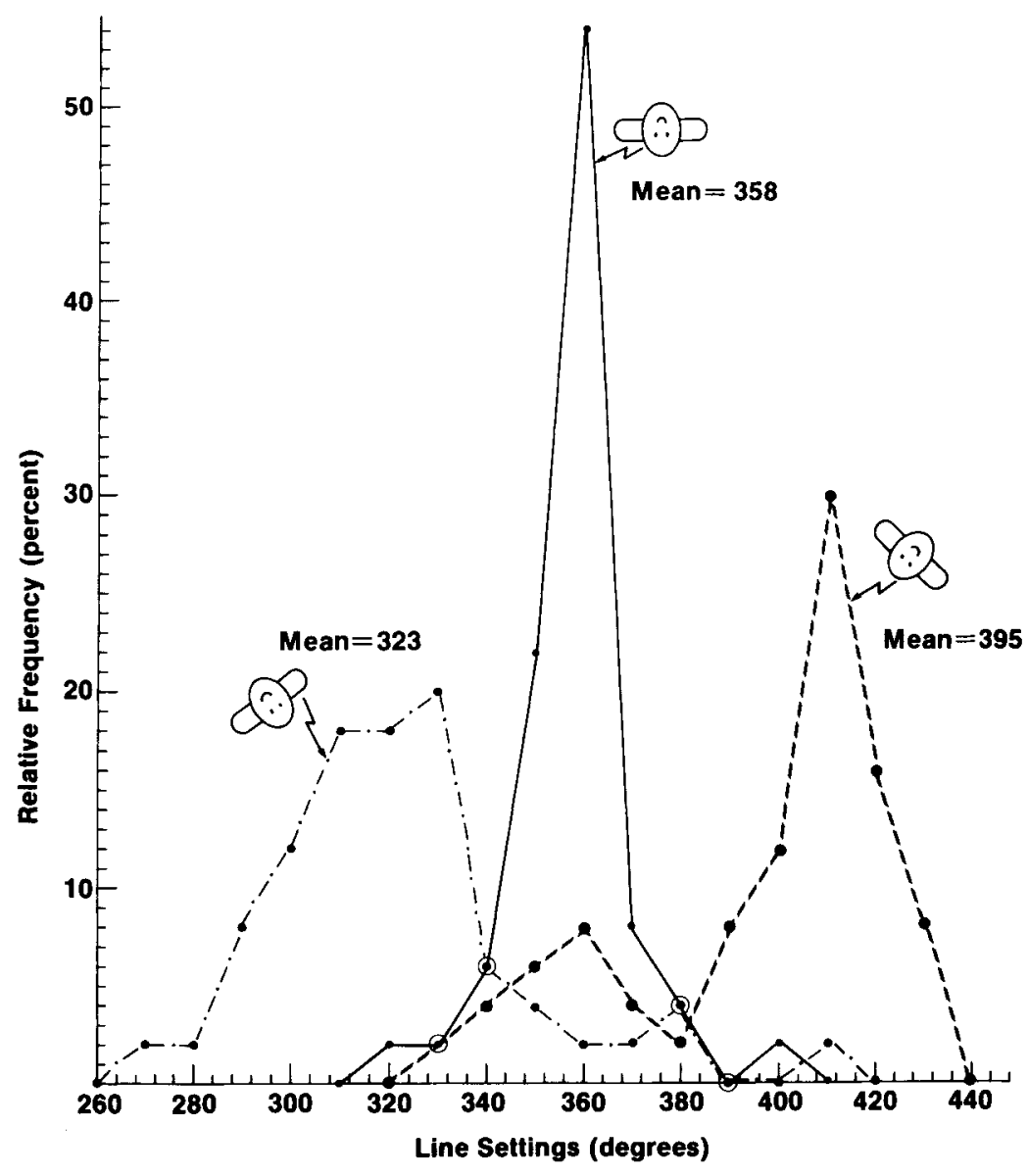

Figure 2. Relative frequency of line-setting values for 50 observers in the straight (head inverted), $30^{\circ}$ left-tilt, and $30^{\circ}$ right-tilt positions. Observers differ in their responses to head tilt from an inverted position.

induced alterations in subjective vertical. Effects due to the body were assessed by keeping the head inverted (straight) while tilting the body ( $\mathrm{S} / \mathrm{T}$, Figure 3 ). The effects due to the head were evaluated by tilting the head while keeping the body straight (X axis vertical) $(T / S)$, and the combined effects of the head and body were determined by tilting both (T/T). Finally, a head-inverted, body-straight $(\mathbf{S} / \mathbf{S})$ position was included to provide a baseline condition.

\section{Method}

Forty-eight undergraduate students served as observers in the third experiment. For each session, the observer set the line twice to his subjective vertical while in each of the four head/body positions. The order of presentation of positions was counterbalanced across observers.

\section{Results}

Figure 3 illustrates ordering of the effects of tilt on subjective vertical, with combined head/body tilt producing the greatest effect and body tilt alone producing the least effect. Analysis of variance indicated that differences in settings as a function of the four positions were significant $[F(3,141)=11.9, p<.0001]$. The average signed error in the settings across observers for the combined head/body tilt condition (T/T) was $36^{\circ}$, very nearly the same as that obtained in the second experiment.

\section{DISCUSSION}

Alternative mechanisms can be proposed to account for the results of the experiments described here. One of these employs the model developed recently by Mittelstaedt (1983). According to this model, visual subjective vertical is calculated from a gravity vector transduced by vestibular and somesthetic receptors summed with vectors representing the orientation of the observer's own head and body axes ("idiotropic vectors"). Visual subjective vertical is determined by the direction of the resultant vector calculated in the brain by summing the idiotropic and gravity vectors. If the gravity vector were assigned a magnitude of 1.0 , the "idiotropic"' head vector a magnitude of 0.4 , and the "idiotropic" body vector a magnitude of 0.2 , the model would predict the following errors in the line settings for Experiment $3: \mathrm{S} / \mathrm{S}, 0^{\circ} ; \mathrm{S} / \mathrm{T}, 13^{\circ} ; \mathrm{T} / \mathrm{S}$, $24^{\circ}$; and T/T, $32^{\circ}$ (see Figure 4 ).

The observers in Experiment 3 exhibited a constant er- 


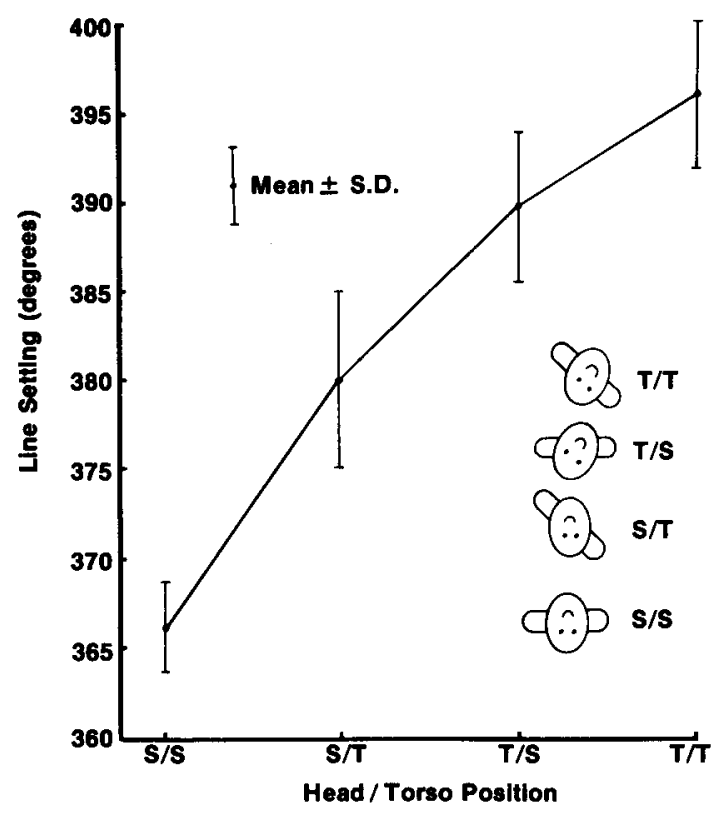

Figure 3. Average subjective vertical values from 48 observers for combined head/and body tilt (T/T), head tilt only (T/S), body tilt only $(S / T)$, and no tilt $(S / S)$. The combination of head and body tilt resulted in the greatest displacement of subjective vertical. ror of about $6^{\circ}$, which may have been produced by a hysteresis effect when they were in the straight $(\mathbf{S} / \mathbf{S})$ position. Summing this constant error with the errors derived from Mittelstaedt's model yields the following predicted line settings for Experiment 3: S/T, $379^{\circ} ; \mathrm{T} / \mathrm{S}, 390^{\circ}$; and $\mathrm{T} / \mathrm{T}, 398^{\circ}$. These predicted line settings are in good agreement with the observed settings: $\mathrm{S} / \mathrm{T}, 379^{\circ} ; \mathrm{T} / \mathrm{S}, 389^{\circ}$; and $\mathrm{T} / \mathrm{T}, 396^{\circ}$. Of course, the magnitude of the predicted errors depends on the magnitudes assigned to the various vectors. The magnitude of 0.4 assigned to the head idiotropic vector derives from Mittelstaedt's calculations based on previous observations. Inclusion in the model of an idiotropic vector representing the observer's ventraldorsal body axis represents an extension of the original Mittelstaedt model, and assignment to this vector of a magnitude of 0.2 was arbitrary.

The finding that some observers reported tilt of subjective vertical in the same direction as head/body tilt is not readily interpreted in the context of Mittelstaedt's model. One possible explanation is that the vestibular and somesthetic receptors do not provide an accurate signal representing the gravity vector. If the gravity vector were rotated clockwise in Figure 4, combinations of the displaced gravity vector with very low magnitudes for the idiotropic vectors could yield predictions for line settings

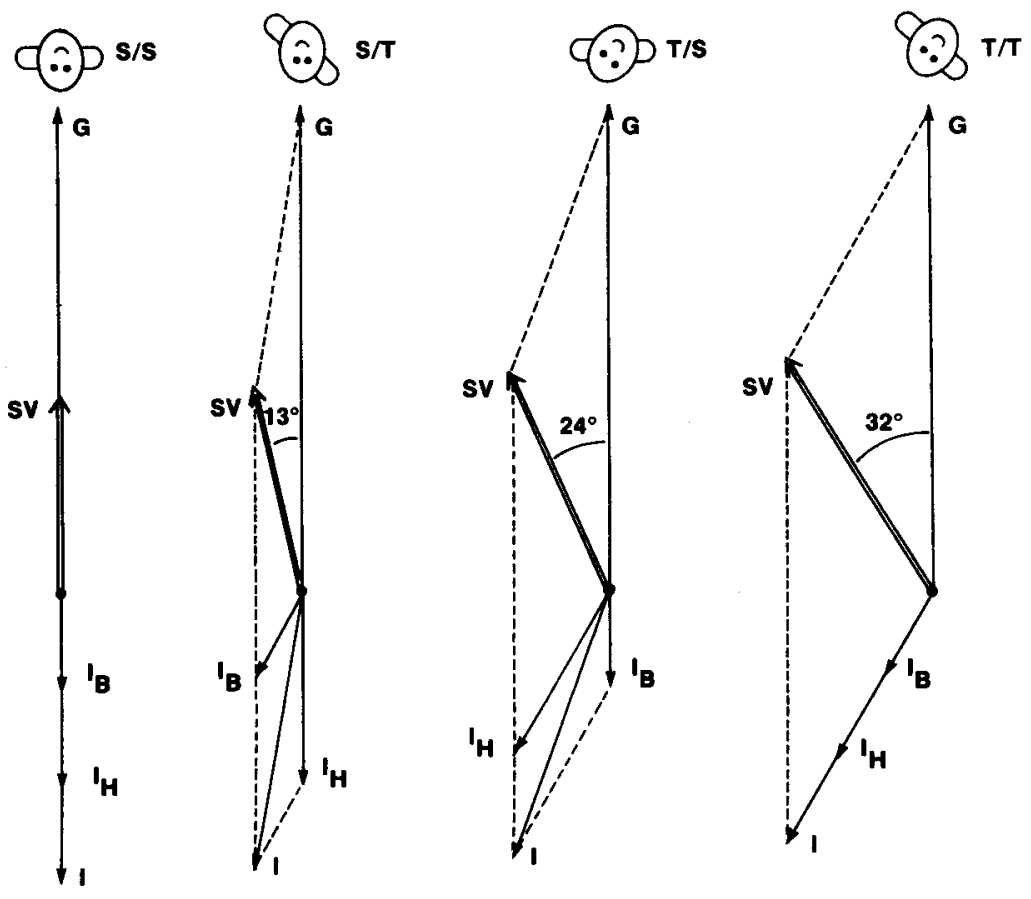

Figure 4. Application of Mittelstaedt's (1983) model to Experiment 3. Subjective vertical (double arrow labeled SV) is the resultant of a vector representing the direction of gravity (G) and an "idiotropic" vector (I) representing the observer's own body axes. The idiotropic vector is the resultant of two components: an idiotropic vector representing the head longitudinal axis $\left(I_{H}\right)$ and an idiotropic vector representing the body ventral-dorsal axis $\left(\mathrm{I}_{\mathrm{B}}\right)$. The model predicts that tilt of the observer's head and body from an inverted position results in tilt of the subjective vertical in the opposite direction. 
in the same direction as the head/body tilts. Mittelstaedt reported the results of one experiment on self-controlled body positioning that suggests consistent errors in the transduction of gravity information. However, the maximum deviations found by him with eight observers were about $5^{\circ}$, which seems insufficient to explain the data from Experiment 2. Further investigation to determine observers' personal constants, both for gravity vector transduction and for idiotropic vector magnitudes, is needed to understand the individual differences found in Experiment 2. Nevertheless, Mittelstaedt's model does provide a very nice explanation of the intuitively nonobvious finding that, for most observers, tilt from a head-inverted position produces displacement of subjective vertical in the direction opposite to the tilt.

An alternative account of our data emphasizes the role of the saccule. It is suggested that the utricular otolith and somesthetic receptors send signals to the brain nuclei that calculate visual orientation. Both the gain and sign of these signals could depend on another signal from the saccular otolith receptors (Schone, 1977; Schone \& Parker, 1967). Individual differences in the responses reported here could be associated with differences in saccular modulation of the utricular and somesthetic signals. However, detailed predictions from and neurophysiological support for this alternative suggestion are lacking.

The results obtained in Experiment 3 are in the same direction as those reported previously (Schone \& Udo de Haes, 1968, 1971). In contrast to our findings, previous data indicated very large variability in line setting within subjects across time. The differences between the two sets of results may be due to differences in procedures. Schone and Udo de Haes started each observation period with the observer in the prone position and then rotated the restraint bed through angles up to $180^{\circ}$. In contrast, the observers in our experiments were placed in the restraint in the supine position and slowly rotated only $30^{\circ}$ to a tilt position. (Our apparatus does not permit us to rotate observers through angles of more than $75^{\circ}$.)

Possible relationships between eye torsion and the changes in subjective visual vertical following tilts from the head-inverted position should be examined. Several investigators were unable to find a relationship between countertorsion and the apparent tilt of a vertical line (see Howard, 1982, p. 430). However, Balliet and Nakayama (1978) reported that voluntarily induced eye torsion was accompanied by shifts of subjective vertical. This suggests that eye torsion may affect subjective vertical differently under different conditions.

The experiments described in this report were undertaken as part of an attempt to identify variables that predict individual differences in motion sickness susceptibility. Forty-eight people from the Johnson Space Center Neu- rophysiology Laboratory subject pool were examined employing the procedure described for Experiment 1. Of this group, 24 had previously exhibited low motion sickness susceptibility during parabolic flight on a NÄSA KC-135 aircraft and the remaining 24 were highly susceptible to the motion sickness produced by the transient periods of weightlessness during this type of flight. Several analyses were performed on the data set for these observers. None revealed a relationship that even approached statistical significance between changes in subjective vertical following tilts from a head-inverted position and either parabolic flight or any of the other provocative motion sickness procedures used at Johnson Space Center. The procedures described in this report do not appear to be useful for predicting motion sickness susceptibility.

\section{REFERENCES}

Balliet, R., \& Nakayama, K. (1978). Egocentric orientation is influenced by trained voluntary cyclorotary movements. Nature, New Biology, 275, 214-216.

BischoF, N. (1974). Optic-vestibular orientation to the vertical. In H. H. Kornhuber (Ed.), Handbook of sensory physiology (Vol. VI/2). Berlin: Springer.

HowARD, I. P. (1982). Human visual orientation. New York: Wiley MittelstaedT, H. (1983). A new solution to the problem of the subjective vertical. Naturwissenschaften, 70, 272-281.

PARKER, D. E. (1980). The vestibular apparatus. Scientific American, 243, 118.

Parker, D. E., Poston, R. L., \& Gulledge, W. L. (1983). Spatial orientation: Visual-vestibular-somatic interaction. Perception \& Psychophysics, 33, 139-146.

Parker, D. E., Wood, D. L., Gulledge, W. L., \& Goodrich, R. L. (1979). Self-motion magnitude estimation during linear oscillation: Changes with head orientation and following fatigue. Aviation Space and Environmental Medicine, 50, 1112-1121.

Schone, H. (1964). On the role of gravity in human spatial orientation. Aerospace Medicine, 35, 764-772.

SCHONE, H. (1975). The "weight" of the gravity organ's signal in the control of perceptual and reflex type orientation at different body positions. Fortstritte der Zoologie, 23, 274-285.

SCHONE, H. (1977). Orientation of perceived vertical as a function of the inputs from statolith organs and somesthetic system. In G. Hauske \& E. Butenandt (Eds.), Kybernetic 1977 (pp. 408-412). Munich: Oldenberg.

Schone, H., \& Parker, D. E. (1967). Inversion of the effect of increased gravity on subjective vertical. Naturwissenschaften, 54, 288-289.

SCHONE, H., \& UdO DE HAEs, H. (1968). Perception of gravity vertical as a function of head and trunk position. Zeitschrift für Vergleichende Physiologie, 60, 440-444.

SCHONE, H., \& Udo DE HAES, H. (1971). Space orientation in humans with special reference to the interaction of vestibular, somesthetic and visual inputs. In H. Drischesl \& N. Tiedt (Eds.), Biokybernetik, Band III (pp. 172-191). Jena: Fischer.

UdO DE HAEs, H., \& SCHONE, H. (1970). Interaction between statolith organs and semicircular canals on apparent vertical and nystagmus. Acta Otolaryngologica, 69, 25-31.

(Manuscript received February 24, 1984; revision accepted for publication September 19, 1984.) 